\title{
Refractive indices of unfilled resin mixtures and cured composites related to color and translucency of conventional and low-shrinkage composites
}

\author{
Vesna Miletic, ${ }^{1}$ Nikola Jakovljevic, ${ }^{1}$ Dragica Manojlovic, ${ }^{1}$ Jovana Marjanovic, ${ }^{1}$ \\ Aleksandra A. Rosic, ${ }^{2}$ Miroslav D. Dramićanin ${ }^{3}$ \\ ${ }^{1}$ University of Belgrade, School of Dental Medicine, DentalNet Research Group, 11000 Belgrade, Serbia \\ ${ }^{2}$ University of Belgrade, Faculty of Mining and Geology, Department of Crystallography, 11000 Belgrade, Serbia \\ ${ }^{3}$ University of Belgrade, Vinča Institute of Nuclear Sciences, 11001 Belgrade, Serbia
}

Received 13 May 2015; accepted 30 August 2015

Published online 15 September 2015 in Wiley Online Library (wileyonlinelibrary.com). DOI: 10.1002/jbm.b.33523

\begin{abstract}
This study correlated the refractive indices (RIs) of unfilled resin mixtures and resin-based composites (RBCs) with color and translucency of conventional and low-shrinkage RBCs. Unfilled resin mixtures based on different ratios of conventional monomers Bisphenol A-glycidyl-methacrylate (BisGMA)/triethyleneglycol-dimethacrylate (TEGDMA) and urethane-dimethacrylate (UDMA)/TEGDMA and a low-shrinkage monomer FIT-852 (FIT, Esstech Inc.)/TEGDMA were used to prepare model RBCs, containing $30 \mathrm{wt} \%$ of the organic matrix and $70 \mathrm{wt} \%$ of silanated barium-glass fillers ( $n=1.553$, Esstech Inc.). The Rls of resins were measured on an ABBE refractometer, those of cured RBCs using the Becke-line method in immersion oils. Color and translucency were determined using an AvaSpec-2048 (Avantes BV) spectrometer. The Rls of unfilled resin mixtures decreased with
\end{abstract}

increasing amounts of TEGDMA. Cured RBCs had higher Rls than their respective resin mixtures. BisGMA-based composites were more translucent with significantly lower $L^{*}$ values than FIT- and UDMA-based RBCs. The RIs of unfilled resins positively correlated with cured RBCs $(p=0.001)$, as did the Rls with translucency $(p=0.001)$ and color $(p=0.008)$. Resin mixtures and corresponding RBCs based on UDMA and its modified low-shrinkage version, FIT, showed similar optical properties. The Rls of unfilled resins appeared to be good predictors of the Rls of cured RBCs. $\odot 2015$ Wiley Periodicals, Inc. J Biomed Mater Res Part B: Appl Biomater, 105B: 7-13, 2017.

Key Words: composite, refractive index, color, translucency, low-shrinkage

How to cite this article: Miletic V, Jakovljevic N, Manojlovic D, Marjanovic J, Rosic AA, Dramicanin MD. 2017. Refractive indices of unfilled resin mixtures and cured composites related to color and translucency of conventional and low-shrinkage composites. J Biomed Mater Res Part B 2017:105B:7-13.

\section{INTRODUCTION}

Contemporary dental composites exhibit a unique balance of esthetic, mechanical, and biological properties aimed at restoring integrity, appearance, and function of hard dental tissues. Their organic-inorganic composition and structure, based on filler particles homogeneously distributed in a resin matrix, do not resemble the composition and structure of natural teeth. Yet, the overall properties of resin-based composites (RBCs) have been continuously developed over the last five decades to reach a point where these materials have become the material of choice for restoring both anterior and posterior teeth. $^{1,2}$

Effects of fillers on the optical properties of RBCs are long known, ever since the introduction of "minifilled" composites in late 1970s. A detailed effect of the type, size, and volume/ mass fraction of fillers on various properties of RBCs was in focus of previous studies. ${ }^{3-6}$ Higher filler volume decreases translucency ${ }^{3}$ and lightness of RBCs almost linearly. ${ }^{4}$ Other color components, chroma and hue, were moderately affected by the amount of fillers in model RBCs. ${ }^{4}$ Salgado et al. ${ }^{5}$ reported on variable effects of nanosized silica fillers on color and translucency of model RBCs. Titanium-dioxide nanoparticles even in minute amounts $(<1 \mathrm{wt} \%)$ showed a significant effect on translucency, color, fluorescence, and opalescence of RBCs. ${ }^{6}$

The effect of monomers on optical properties of RBCs, mainly translucency, is associated with the refractive index (RI) of the monomer phase and its relation to the refractive index of the filler phase. ${ }^{7,8}$ It is generally accepted that the greater the mismatch between the RIs of fillers and monomers, the lower the translucency of the cured RBC. It was also shown that the RI of uncured material changes upon curing ${ }^{8,9}$ or certain clinical treatments, such as bleaching. ${ }^{10}$ Shortall et al. ${ }^{8}$ suggested that better shade-matching between RBCs and teeth could be obtained if the RI difference between the monomer and filler phase was equal before and after curing. Other properties such

Correspondence to: V. Miletic; e-mail: vesna.miletic@stomf.bg.ac.rs

Contract grant sponsor: Ministry of Education, Science and Technological Development, Republic of Serbia; contract grant number: ON172007, ON176016, 45020 
as opacity, color characteristics, and surface gloss were also correlated with the RI of experimental RBCs. Translucency decreased whilst opacity and surface gloss increased with increased RI of composites. ${ }^{11}$ A recent study on nanogel prepolymers added to model RBCs showed increased RI and lower translucency of monomer/nanogel formulations. ${ }^{12}$

Low-shrinkage RBCs were developed to overcome the single most unfavorable property of RBCs, that is, polymerization shrinkage and the associated stress. ${ }^{2,13-17}$ These RBCs are used as posterior teeth restoratives and as such are expected to possess at least satisfactory optical and esthetic properties. However, literature lacks data on optical properties of low-shrinkage RBCs and especially the effect of RI of low-shrinkage monomers on optical properties of their respective RBCs. One reason could be the focus of manufacturers and researchers on mechanical properties, curing kinetics, shrinkage, and shrinkage stress. Another reason might be that most of the low-shrinkage monomers in commercial low-shrinkage RBCs are patent protected and, thus, unavailable to independent researchers.

The aim of the study was to evaluate the effect of RIs of unfilled resin mixtures and cured RBCs on color and translucency of model conventional and low-shrinkage RBCs. The null hypotheses were: (1) there is no difference in color and translucency between model RBCs; (2) there is no correlation between the RIs of unfilled resin mixtures and cured RBCs; (3) there is no correlation between the RIs of resin mixtures/RBCs and color/translucency of model RBCs.

\section{MATERIALS AND METHODS}

\section{Preparation of unfilled resin mixtures and RI} measurements

Conventional monomers bisphenol A-glycidyl methacrylate (BisGMA), urethane dimethacrylate (UDMA), triethyleneglycoldimethacrylate (TEGDMA) from Sigma-Aldrich Chemie GmbH, Munich, Germany and a low-shrinkage monomer FIT-852 (FIT) resin from Esstech Inc., Essington, PA, USA were used to prepare the following resin mixtures (ratio by weight):

BisGMA:TEGDMA-3:1, 2:1, 1:1, 1:2, 1:3

UDMA: TEGDMA-3:1, 2:1, 1:1, 1:2, 1:3

FIT:TEGDMA-3:1, 2:1, 1:1, 1:2, 1:3

Resin mixtures were prepared by measuring about $2 \mathrm{~g}$ of BisGMA, UDMA, and FIT in a light-free test tube on an analytical balance $(d=0.01$, ACCULAB ALC-110.4, Sartorius group, Goettingen, Germany) and adding the appropriate amount of TEGDMA to produce the above stated weight ratio in each group. Resins were homogenized on a rotator at $20 \mathrm{rpm}$ for $12 \mathrm{~h}$. The RIs of each resin and their mixtures were measured on an ABBE refractometer (EUInstruments) at $21.7 \pm 0.8^{\circ} \mathrm{C}$. One drop of resin or resin mixture was placed with a Pasteur pipette on the refractive prism, the dark/light line was brought to the center of the viewing space, and the RI value was noted from the scale.

Preparation of model composites and RI measurements BisGMA:TEGDMA, UDMA:TEGDMA, and FIT:TEGDMA resin mixtures based on 2:1 and 1:1 monomer ratios were used to prepare model RBCs. The photoinitiator system, camphorquinone and $N, N$-dimethylaminoethylmethacrylate (both Sigma-

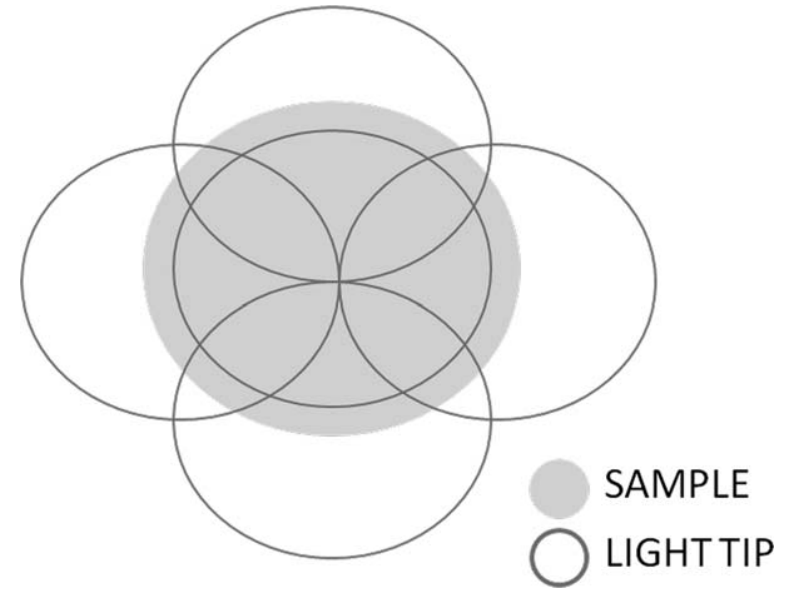

FIGURE 1. Curing scheme according to the ISO 4049:2009 standard. Each sample was irradiated five times, $40 \mathrm{~s}$ each irradiation, at 12-36-9 o'clock positions and ending in the center of the sample.

Aldrich Chemie $\mathrm{GmbH}$ ), was added in the amount of 0.5 wt \% each, relative to the resin mixture, and ultrasonicated for 1 min to dissolve. Silanated barium-glass fillers (Esstech Inc, average size $0.7 \mu \mathrm{m}, \mathrm{RI}=1.553$ ) were added to the organic mixture sequentially and mechanically stirred in light-free conditions. Final model RBCs consisted of $70 \mathrm{wt} \%$ of inorganic fillers and $30 \mathrm{wt} \%$ of the organic matrix.

Stainless steel molds, $18 \mathrm{~mm}$ in diameter and $2 \mathrm{~mm}$ thick, were placed on a glass slab filled with composite and pressed with a glass slide to extrude excess material. Each sample ( $n=3$ per group) was light-cured using an LED light-curing unit (LEDition, Ivoclar Vivadent, Schaan, Liechtenstein) at an intensity of around $800 \mathrm{~mW} / \mathrm{cm}^{2}$, held against the glass slide to maintain distance. The curing scheme was adopted from the ISO 4049:2009 standard (Figure 1). ${ }^{18}$ Upon curing, the samples were kept dry at $37^{\circ} \mathrm{C}$ for $24 \mathrm{~h}$ prior to further measurements.

The RIs of cured and milled RBCs were measured by an immersion method (Becke-line method) at room temperature using a polarized-light microscope (REICHERT NEOVAR-POL, Mikrovid GmbH, Arnsberg, Germany) and Cargille Immersion set (Cargille Laboratories, Cedar Grove, NJ, USA). According to data from Cargille liquids for temperature coefficient $-\mathrm{dRI} / \mathrm{d} T$, the RI values were corrected.

\section{Measurements of translucency and color}

Translucency and color were measured using an AvaSpec2048 spectrometer (Avantes BV, Apeldoorn, The Netherlands), operating in the transmittance mode, against a black and white background. CIELab coordinates were calculated from reflection spectra for the standard illuminant D65. Translucency parameter (TP) was calculated according to the following equation:

$$
\mathrm{TP}=\sqrt{(L 1-L 2)^{2}+(a 1-a 2)^{2}+(b 1-b 2)^{2}}
$$

where $L 1$ and $L 2, a 1$ and $a 2, b 1$ and $b 2$ are the CIELab values against the black and white background, respectively. 


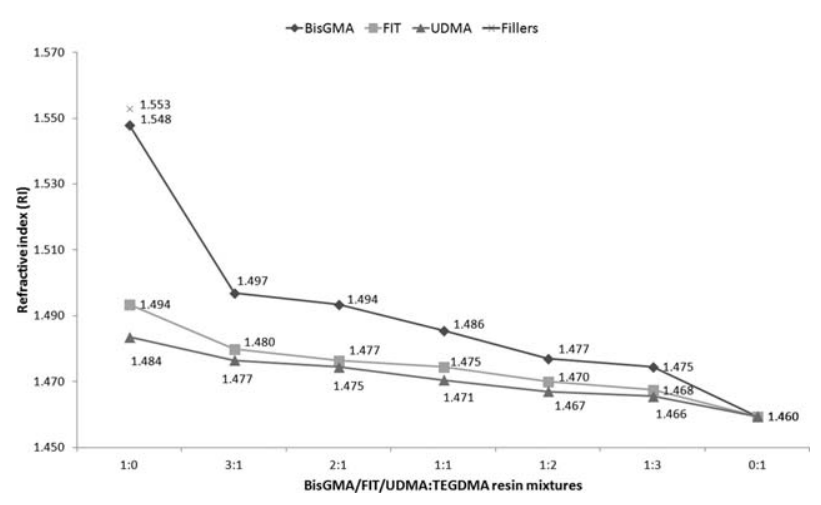

FIGURE 2. The RIs of unfilled resin mixtures based on different monomer ratios.

Color deviation from "ideal white" $(\Delta E)$ of each RBC was calculated using the following equation:

$$
\Delta E=\sqrt{\Delta L^{2}+\Delta a^{2}+\Delta b^{2}}
$$

where $\Delta L, \Delta a$, and $\Delta b$ were calculated as:

$\Delta L=L^{*}$ sample- $L^{*}$ standard for "ideal white" (100); $\Delta a=a^{*}$ sample- $a^{*}$ standard for "ideal white" (0), $\Delta b=b^{*}$ sample- $b^{*}$ standard for "ideal white" $(0)$.

\section{Statistical analysis}

Data were statistically analyzed in Minitab 16 (Minitab Inc. State College, PA, USA). One-way analysis of variance (ANOVA) with Tukey's post hoc test was used to test the differences between TP and $\Delta E$ values of cured RBCs. Pearson's correlation coefficient and regression analysis were used to examine the correlation between the RIs of unfilled resins/cured RBCs and optical parameters of model RBCs. The level of significance was set at $\alpha=0.05$.

\section{RESULTS}

Figure 2 presents the RIs of unfilled resin mixtures based on different ratios of base monomers. The RIs of BisGMA: TEGDMA were closer to the RI of filler particles (1.553) than the RIs of UDMA:TEGDMA and FIT:TEGDMA resin mixtures. An increase of TEGDMA and decrease of base monomer in

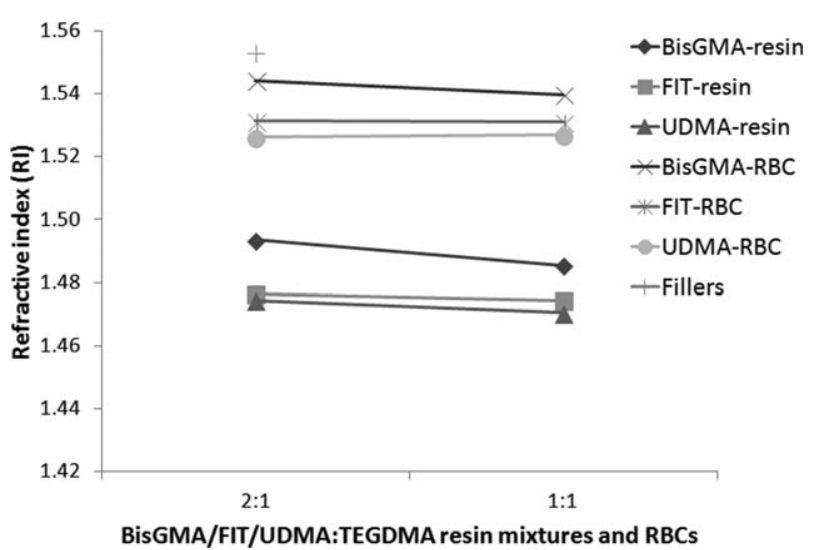

FIGURE 4. The RIs of cured RBCs compared to the RIs of unfilled resin mixtures.

resin mixtures decreased the RIs in all groups but to a different extent. The RIs of resin mixtures used in RBCs were in the range of 1.474 (UDMA:TEGDMA) and 1.493 (BisGMA:TEGDMA) for 2:1 ratio mixtures and 1.470 (UDMA:TEGDMA) and 1.485 (BisGMA: TEGDMA) for 1:1 ratio mixtures.

Representative images of cured and milled RBC particles prepared for RI measurements are presented in Figure 3. Inorganic filler and organic resin matrix appeared as irregular-shaped, homogeneous particles 50-500 $\mu \mathrm{m}$ in diameter. Therefore, the RI of the cured RBC relates to the composite as a whole and not to the either phase (filler or resin) within the polymer.

The RIs of cured RBCs are compared to those of unfilled resins in Figure 4. In all groups, the RIs increased upon curing of RBCs and approached the RI of pure filler particles. Similarly to unfilled resins, BisGMA-based RBCs exhibited closer RIs to that of the filler particles (1.553) compared to FIT- and UDMA-based RBCs. An increase of TEGDMA and decrease of base monomer (BisGMA, FIT and UDMA) resulted in lower RIs of BisGMA- (1.544-1.540) and FIT-based RBCs (1.5321.531). Conversely, a slight increase in the RI of UDMA-based RBCs (1.526-1.527) was found with the increased amount of TEGDMA.

Figure 5 presents $\Delta E$ and TP values for the cured RBCs. BisGMA-based RBCs showed significantly higher $\Delta E$ and TP
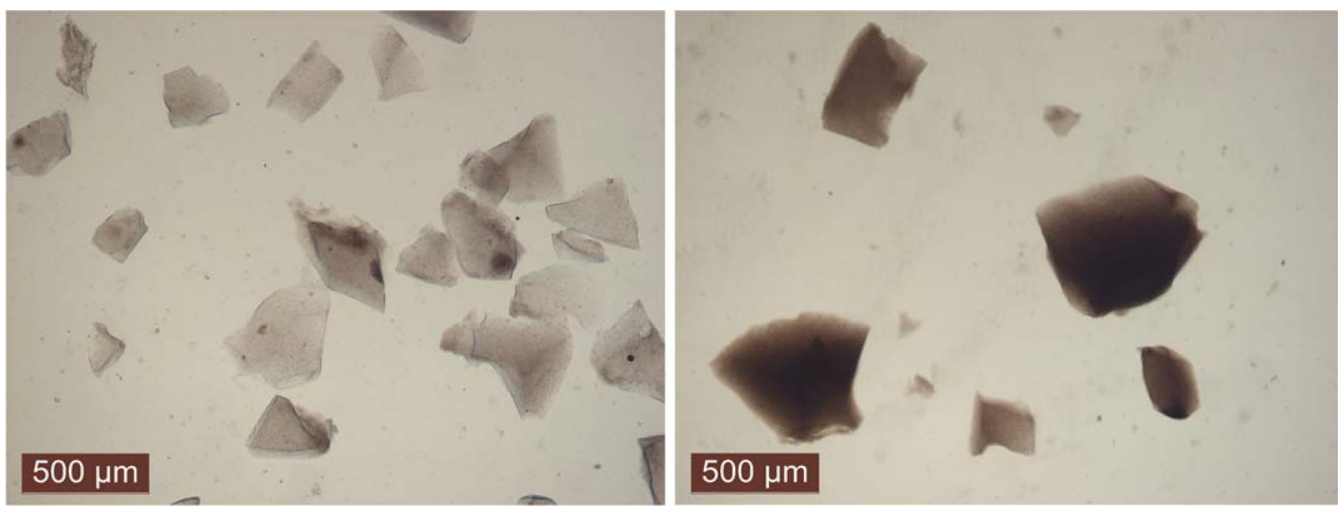

FIGURE 3. Representative images of milled particles of cured RBCs prepared for RI measurements using the Becke-line method. BisGMA: TEGDMA 1:1 (left), UDMA:TEGDMA 1:1 (right). 


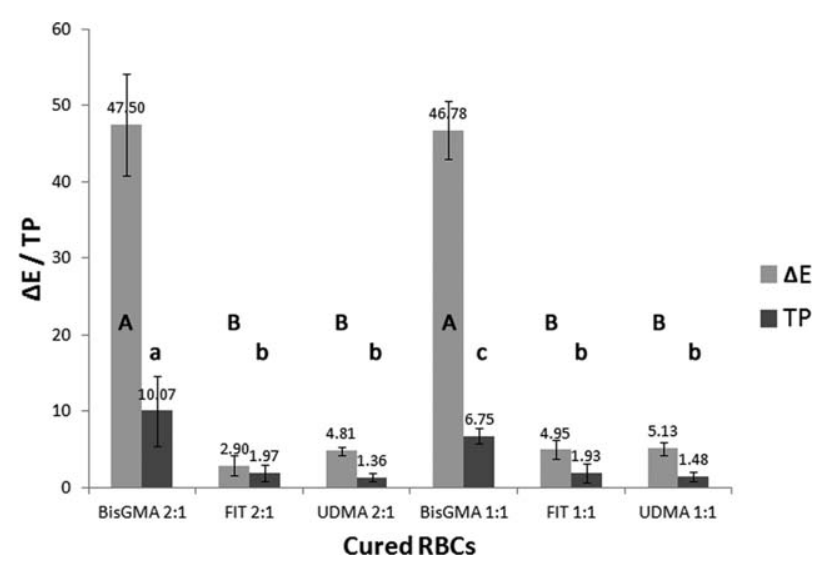

FIGURE 5. Color and translucency of the cured RBCs based on 2:1 or 1:1 BisGMA/UDMA/FIT to TEGDMA ratio resin mixtures. Color is expressed as the deviation from "ideal white." Groups that share the same letter are not significantly different $(p>0.05)$.

values than FIT- and UDMA-based RBCs $(p<0.05)$. BisGMAbased RBC based on 2:1 BisGMA:TEGDMA ratio had significantly higher TP than its counterpart based on 1:1 BisGMA:TEGDMA ratio. FIT- and UDMA-based RBCs had similar $\Delta E$ and TP values $(p>0.05)$. There was a trend that BisGMAand FIT-based RBCs with lower amounts of TEGDMA (2:1) had higher TP than their counterparts with higher TEGDMA amount (1:1). Conversely, a lower amount of TEGDMA in the UDMA-based RBC was associated with slightly lower mean TP, as in the case of RIs. Increasing TEGDMA in FITand UDMA-based RBCs increased $\Delta E$ albeit the difference was not significantly different.

Table I presents mean values of the CIELab coordinates for the tested RBCs. Indicating lightness, CIE $L^{*}$ of BisGMAbased RBCs was nearly half the value of those for UDMAand FIT-based RBCs. CIE $a^{*}$ and CIE $b^{*}$ indicating red-green and yellow-blue coordinates, respectively, were higher in BisGMA-based RBCs.

Correlation and regression analyses indicated strong correlation between the RIs of unfilled resin mixtures and the RIs of cured RBCs (Figure 6). A higher RI of unfilled resin mixtures resulted in a higher RI of the cured RBC. Furthermore, positive correlation was found between the RIs of both unfilled resin mixtures and cured RBCs and TP values (Figure 7). Somewhat stronger correlation existed between the RIs of unfilled resin mixtures and TP. The RIs of both unfilled resin mixtures and cured RBCs positively correlated

TABLE I. Mean CIELab Values of RBCs Based on Different Monomer Ratios

\begin{tabular}{lrrr}
\hline & \multicolumn{3}{c}{ Mean } \\
\cline { 2 - 4 } RBC & \multicolumn{1}{c}{$L^{*}$} & $a^{*}$ & $b^{*}$ \\
\hline BisGMA:TEGDMA 2:1 & 52.59 & 0.84 & 1.64 \\
FIT:TEGDMA 2:1 & 100.75 & 1.24 & 1.06 \\
UDMA:TEGDMA 2:1 & 95.40 & 0.28 & 1.20 \\
BisGMA:TEGDMA 1:1 & 53.46 & 2.42 & 3.94 \\
FIT:TEGDMA 1:1 & 95.11 & 0.38 & 0.53 \\
UDMA:TEGDMA 1:1 & 94.92 & 0.46 & 0.49 \\
\hline
\end{tabular}
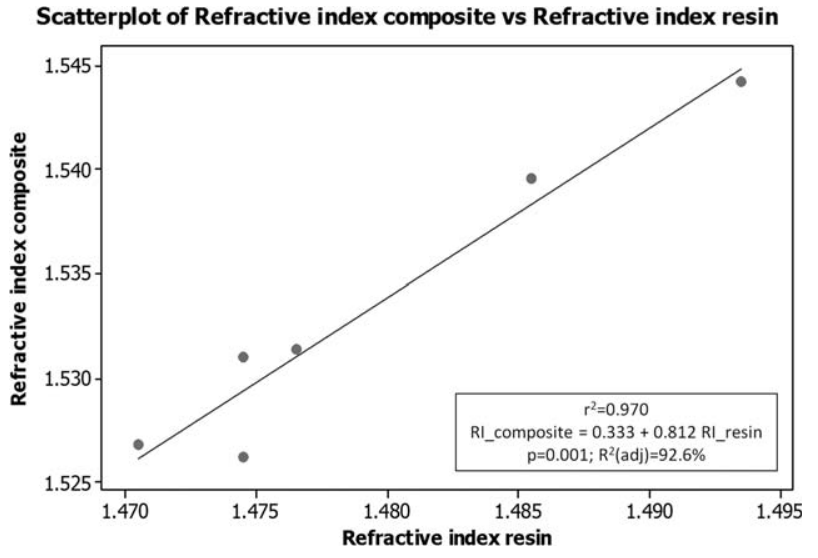

FIGURE 6. Scatterplot of the RIs of unfilled resin mixtures and cured RBCs with the Pearson's correlation coefficient $\left(r^{2}\right)$, regression equation, and $p$ values indicating statistical signficance. $R^{2}(\operatorname{adj})$ estimates the strength of the relationship i.e. describes the amount of variation in $\mathrm{RI}$ _composite that is explained by $\mathrm{RI}$ resin.

with $\Delta E$ values (Figure 8). However, $p$ and $R^{2}$ (adj) values indicate a weaker relationship between the RIs and $\Delta E$ compared to that of RIs and TP.

\section{DISCUSSION}

Significant differences were found between the tested model RBCs. Positive correlations were established between the RIs of unfilled resin mixtures and filled polymer, that is, cured RBCs as well between the RIs and optical properties of RBCs. Therefore, all three null hypotheses were rejected.

In the present study, a low-shrinkage monomer FIT was used to prepare a low-shrinkage RBC. According to the manufacturer (Esstech Inc.) it is a non-linear, bifunctional urethanebased methacrylate with a molecular weight between 1100 and 1200. A higher degree of conversion, lower shrinkage, and better flexural strength of FIT-based than BisGMA-based experimental RBCs were previously reported. ${ }^{19}$ In the present study, FIT was used as a model low-shrinkage monomer instead of patent protected urethane-based monomers found in commercial RBCs, such as Venus Diamond (Heraeus Kulzer) and GC Kalore (GC Corp.).

The results revealed higher RIs of BisGMA- than UDMA- and FIT-based mixtures and consequently higher RIs of cured RBCs based on these resin mixtures. A previous study also found higher RIs upon curing of RBCs containing UDMA and TEGDMA monomers and colloidal silica fillers despite the fact that the RI of silica was lower than that of unfilled, uncured resins. ${ }^{9}$ Shortall et al. ${ }^{8}$ reported increased RIs post-polymerization for a series of BisGMA:TEGDMA RBCs containing barium or strontium-glass fillers, in most groups exceeding the RIs of pure filler particles. In the present study, the RIs of cured RBCs did not exceed the RI of filler particles. The difference between the two studies could be attributed to the fact that barium-glass fillers with higher RIs were used in the present study (1.553) compared to the fillers used in the study by Shortall et al. ${ }^{8}$

There was a strong positive correlation between the RIs of unfilled resin mixtures and cured RBCs. This finding indicates the possibility to predict the RI of a cured RBC based 


\section{Scatterplot of Translucency vs Refractive index}

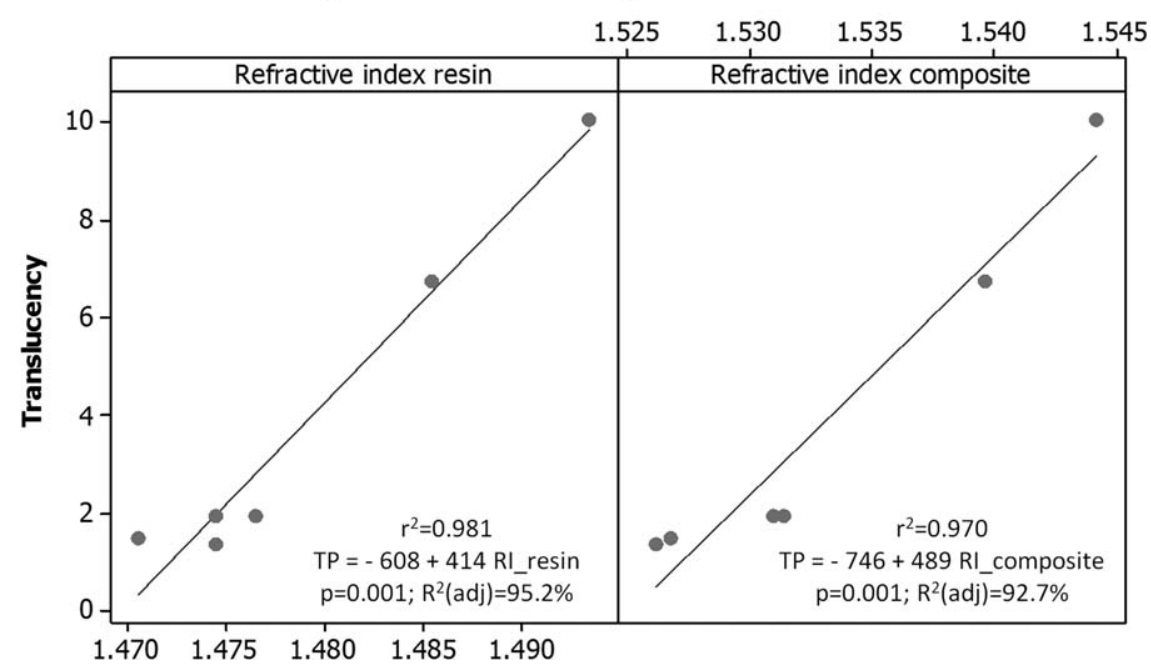

FIGURE 7. Scatterplot of TP values related to the RIs of unfilled resin mixtures and cured RBCs with Pearson's correlation coefficient $\left(r^{2}\right)$ and the summary of regression analysis.

on the RI of its major crosslinking or base monomers. Measurement of the RIs of unfilled resins using a simple ABBE refractometer is a lot easier and less time-consuming as they are all transparent liquids than sample preparation of cured RBCs for the Becke-line method based on a series of immersion oils.

In all unfilled resin mixtures, the RIs decreased with decreased amounts of base monomer, BisGMA, FIT, or UDMA and increased amount of diluent monomer, TEGDMA. This was followed by decreased RIs of corresponding BisGMA- and FIT-based RBCs. A similar finding was reported for unfilled uncured and cured BisGMA:TEGDMA polymer containing different monomer ratios. $^{20}$ Conversely, UDMA-based RBC showed slightly increased RI for 1:1 than 2:1 resin mixture, that is, an increased amount of TEGDMA resulted in an increased RI of the cured RBC. The same trend was observed for TP values of UDMA-based RBCs. The RI seems to be the major contributor to translucency of RBCs as it strictly followed the RI changes in all tested groups. Even in the UDMAbased RBCs exhibiting a different RI trend than other groups, TP values were also atypical, that is, higher with a decreased amount of UDMA and increased amount of TEGDMA.

Significantly higher translucency of BisGMA- than UDMAbased RBCs corresponded well to the study by Azzopardi et al. ${ }^{7}$ despite the fact that these authors compared a 2:1 BisGMA:TEGDMA-based RBC to a pure UDMA-based RBC using a different measuring equipment. A similar finding for two different studies indicates a strong influence of BisGMA on composite translucency irrespective of monomer ratios. This strong influence of BisGMA may be associated with its higher RI

\section{Scatterplot of DeltaE vs Refractive index}

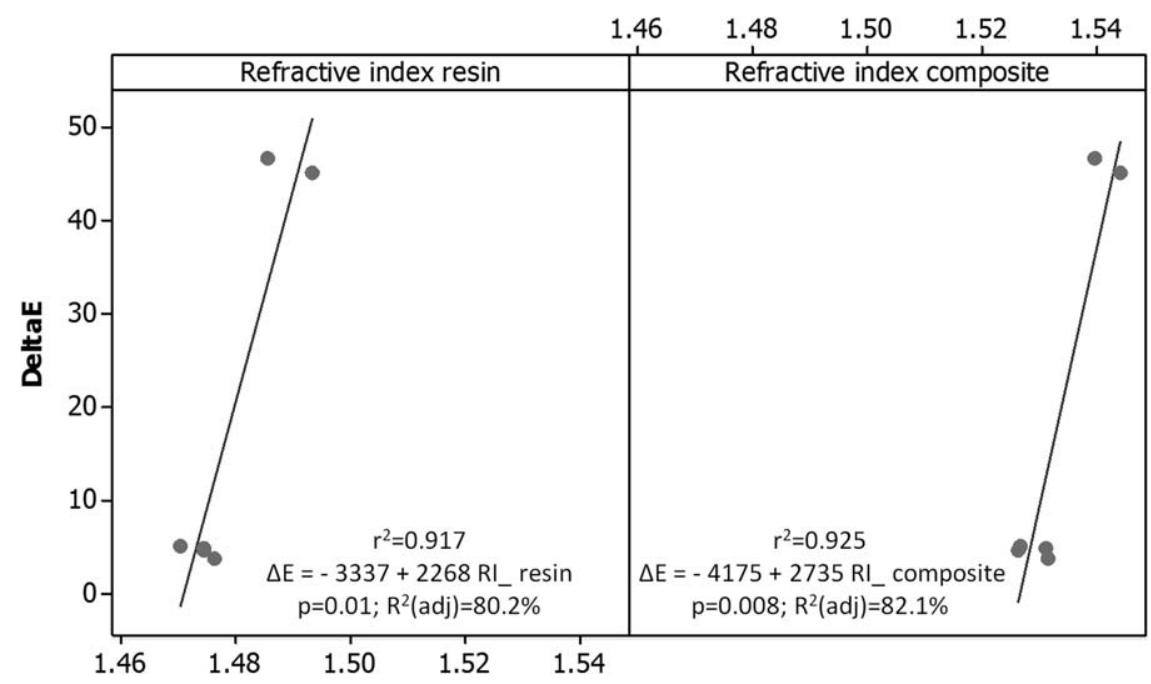

FIGURE 8. Scatterplot of $\Delta E$ values related to the Rls of unfilled resin mixtures and cured RBCs with Pearson's correlation coefficient $\left(r^{2}\right)$ and the summary of regression analysis. 
compared to other commonly used monomers, UDMA and TEGDMA. The present results confirmed higher RIs of unfilled BisGMA-based resin mixtures and cured RBCs compared to those based on UDMA and its modified version, that is, urethane-based low-shrinkage monomer FIT. Despite the fact that FIT has $2.3-2.5 \times$ greater molecular weight than UDMA, the two monomers have shown similar optical properties in unfilled and filled materials.

BisGMA-based RBCs exhibited around 10-fold greater color deviation from "ideal white" compared to UDMA- and FIT-based RBCs. The grayish color of BisGMA-based RBCs was mainly determined by the $L^{*}$ coordinate and to a much lesser extent by the $b^{*}$ coordinate. Lightness $\left(L^{*}\right)$ varied to a great extent even in commercial RBCs, ${ }^{21}$ but obviously not as much as in model composites whose optical properties are not modified by pigments.

A better fitted relationship was found between the RIs of unfilled resin and translucency of RBCs than between the RIs of the RBCs and their translucency. RBCs present more complex and heterogeneous materials with potentially greater micro-structural variation than unfilled resins which are homogeneously transparent. The presence of fillers not only influences the RIs and translucency but also other optical parameters of RBCs, such as opacity, light scattering, reflectivity, and absorption., ${ }^{3,22}$ Lee performed multiple regression analyses to establish potential predictors among the optical properties and color coordinates of cured RBCs and reported variable coefficients (0.723-0.994). ${ }^{22}$

Color of cured RBCs and the RIs showed a weaker fit than translucency and the RIs. Though $\Delta E$ values were not significantly different between FIT- and UDMA-based RBCs, the mean values did not quite follow the trend for the RIs. As the RIs of cured RBCs are seldom measured in the literature, no similar data were found for comparison. Hubbezoglu et al. ${ }^{10}$ measured the RIs of RBCs before and after bleaching but made no correlation with the initial color.

Great differences in translucency and color parameters between the cured RBCs indicate to what extent manufacturers need to modify the final optical parameters of their materials. The final optical properties of a dental restoration are not only influenced by material optical parameters which may change during the course of polymerization, ${ }^{8,23-25}$ but also by layer thickness. ${ }^{26-28}$ In case of highly translucent BisGMA-based RBCs, thick layers in anterior teeth, unsupported by opaque dentin layers often result in an unwanted grayish shade of the final restoration. The grayish shade of BisGMA-based RBCs was confirmed in the present study as substantially greater deviation from "ideal white" compared to UDMA- and FIT-based RBCs.

\section{CONCLUSION}

Higher RIs of unfilled BisGMA:TEGDMA resin mixtures and RBCs were associated with higher translucency and greater deviation from "ideal white" of BisGMA-based RBCs compared to UDMA- and FIT-based materials. Resin mixtures and their corresponding RBCs based on UDMA and its modified lowshrinkage version, FIT, showed similar optical properties, the
RIs, translucency, and color. The RIs of unfilled resins appeared to be good predictors of the RIs of the filler polymer. Both the RIs of unfilled resins and RBCs positively correlated with translucency and, to a lesser extent, color of model RBCs. Manufacturers of commercial low-shrinkage RBCs should provide information on the RIs of their low-shrinkage monomers and resin mixtures to allow understanding of the optical and esthetic performance of such RBCs.

\section{ACKNOWLEDGMENT}

The authors are grateful to Esstech Inc, Essington, PA, USA for a generous donation of FIT-852 resin and Ba-glass fillers used in the study.

\section{REFERENCES}

1. Ferracane JL. Resin composite-State of the art. Dent Mater 2011; 27:29-38.

2. Cramer NB, Stansbury JW, Bowman CN. Recent advances and developments in composite dental restorative materials. J Dent Res 2011;90:402-416.

3. Lee YK. Influence of filler on the difference between the transmitted and reflected colors of experimental resin composites. Dent Mater 2008;24:1243-1247.

4. Lim YK, Lee YK, Lim BS, Rhee SH, Yang HC. Influence of filler distribution on the color parameters of experimental resin composites. Dent Mater 2008;24:67-73.

5. Salgado VE, Albuquerque PP, Cavalcante LM, Pfeifer CS, Moraes RR, Schneider LF. Influence of photoinitiator system and nanofiller size on the optical properties and cure efficiency of model composites. Dent Mater 2014;30:e264-e271.

6. Yu B, Ahn JS, Lim Jl, Lee YK. Influence of TiO2 nanoparticles on the optical properties of resin composites. Dent Mater 2009;25:1142-1147.

7. Azzopardi N, Moharamzadeh K, Wood DJ, Martin N, van Noort R. Effect of resin matrix composition on the translucency of experimental dental composite resins. Dent Mater 2009;25:1564-1568.

8. Shortall AC, Palin WM, Burtscher P. Refractive index mismatch and monomer reactivity influence composite curing depth. J Dent Res 2008;87:84-88.

9. Fujita K, Nishiyama N, Nemoto K, Okada T, Ikemi T. Effect of base monomer's refractive index on curing depth and polymerization conversion of photo-cured resin composites. Dent Mater J 2005; 24:403-408.

10. Hubbezoglu I, Akaoglu B, Dogan A, Keskin S, Bolayir G, Ozcelik S, Dogan OM. Effect of bleaching on color change and refractive index of dental composite resins. Dent Mater J 2008;27:105-116.

11. Ota M, Ando S, Endo H, Ogura Y, Miyazaki M, Hosoya Y. Influence of refractive index on optical parameters of experimental resin composites. Acta Odontol Scand 2012;70:362-367.

12. Moraes RR, Garcia JW, Barros MD, Lewis SH, Pfeifer CS, Liu J, Stansbury JW. Control of polymerization shrinkage and stress in nanogel-modified monomer and composite materials. Dent Mater 2011;27:509-519.

13. Hirata R, Clozza E, Giannini M, Farrokhmanesh E, Janal M, Tovar N, Bonfante EA, Coelho PG. Shrinkage assessment of low shrinkage composites using micro-computed tomography. J Biomed Mater Res B Appl Biomater 2015;103:798-806.

14. Ilie N, Jelen E, Clementino-Luedemann T, Hickel R. Low-shrinkage composite for dental application. Dent Mater J 2007;26:149-155.

15. Akbarian G, Ameri H, Chasteen JE, Ghavamnasiri M. Fracture resistance of premolar teeth restored with silorane-based or dimethacrylate-based composite resins. J Esthet Restor Dent 2014;26:200-207.

16. Boroujeni PM, Mousavinasab SM, Hasanli E. Effect of configuration factor on gap formation in hybrid composite resin, lowshrinkage composite resin and resin-modified glass ionomer. J Investig Clin Dent 2015;6:156-160.

17. Liu WW, He XP, Mo AC, Yao QQ, Ye J, Jing N. Investigation of the mechanical properties of a low-shrinkage liquid crystalline matrix combined with nano-hydroxyapatite. Int J Nanomedicine 2011;6:1787-1791. 
18. ISO 4049. Dentistry-Polymer-based restorative materials. 2009. International Organization for Standardization. Geneva, Switzerland.

19. Johnston A, Rueggeberg FA, Rawls HR, Slaff $H$, Barclift $T$, Duff J. Physical properties of new low-shrink resin. J Dent Res 2010;89: 2295.

20. Lehtinen J, Laurila T, Lassila LV, Vallittu PK, Raty J, Hernberg R. Optical characterization of bisphenol-A-glycidyldimethacrylatetriethyleneglycoldimethacrylate (BisGMA/TEGDMA) monomers and copolymer. Dent Mater 2008;24:1324-1328.

21. Mikhail SS, Schricker SR, Azer SS, Brantley WA, Johnston WM. Optical characteristics of contemporary dental composite resin materials. J Dent 2013;41:771-778.

22. Lee YK. Influence of scattering/absorption characteristics on the color of resin composites. Dent Mater 2007;23:124-131.

23. Celik EU, Aladag A, Turkun LS, Yilmaz G. Color changes of dental resin composites before and after polymerization and storage in water. J Esthet Restor Dent 2011;23:179-188.
24. Domingos PA, Garcia PP, Oliveira AL, Palma-Dibb RG. Composite resin color stability: Influence of light sources and immersion media. J Appl Oral Sci 2011;19:204-211.

25. Friebel M, Pernell O, Cappius HJ, Helfmann J, Meinke MC. Simulation of color perception of layered dental composites using optical properties to evaluate the benefit of esthetic layer preparation technique. Dent Mater 2012;28:424-432.

26. Ferraris F, Diamantopoulou S, Acunzo R, Alcidi R. Influence of enamel composite thickness on value, chroma and translucency of a high and a nonhigh refractive index resin composite. Int $\mathrm{J}$ Esthet Dent 2014;9:382-401.

27. Kamishima N, Ikeda T, Sano H. Effect of enamel shades on color of layered resin composites. Dent Mater J 2006;25:26-31.

28. Khashayar G, Dozic A, Kleverlaan CJ, Feilzer AJ, Roeters J. The influence of varying layer thicknesses on the color predictability of two different composite layering concepts. Dent Mater 2014;30: 493-498. 